\title{
ITER ECE: PLANS AND CHALLENGES
}

\author{
M.E. AUSTIN, P.E. PHILLIPS, W.L. ROWAN \\ University of Texas Fusion Research Center \\ Austin, TX 78712, USA \\ J. BENO, H-P. LIU, A. OUROUA \\ University of Texas Center for Electromechanics \\ Austin, TX 78758, USA
}

\section{R.F. ELLIS}

University of Maryland College Park, MD 20742, USA

\author{
R.W. HARVEY \\ CompX \\ Del Mar, CA 92014, USA \\ A.E. HUBBARD \\ Massachusetts Institute of Technology \\ Cambridge, MA 02139, USA \\ G. TAYLOR, D.W. JOHNSON \\ Princeton Plasma Physics Laboratory \\ Princeton, NJ 08543, USA
}

\begin{abstract}
Measuring ECE in ITER plasmas will present several difficulties in both hardware and physics that will impede efforts to determine $T_{\mathrm{e}}(\mathrm{r}, \mathrm{t})$ and characteristics of the electron distribution function. Recent work on the diagnostic systems and techniques to be employed on ITER has clarified some of these issues and found some solutions. Studies show that even with the greater relativistic broadening due to the high electron temperature, $T_{\mathrm{e}}$ measurements with spatial resolution of 6-10 $\mathrm{cm}$ are still possible especially with properly designed front end optics and instruments. ECE will still be able to provide high resolution $T_{\mathrm{e}}$ profiles in both core and edge regions and even follow oscillations of high $m, n$ TAE modes. Of greater concern is the possibility and effects of non-Maxwellian electron distributions created by intense auxiliary heating. In these cases modeling will have to be used to correct and define the valid limits of $T_{\mathrm{e}}$ from ECE and a special oblique viewing antenna is recommended. The planned ECE instruments, heterodyne radiometer and Michelson interferometer, will provide complementary
\end{abstract}


measurement capabilities. A viable in-port-plug calibration source for these instruments is a concern; progress on work being done to develop a prototype is reported.

\section{Introduction}

This paper presents some of the latest information on plans for the Electron Cyclotron Emission (ECE) diagnostic systems to be installed on ITER. The last publications detailing the ECE diagnostics on ITER were a paper published in 2001 [1] and the Design Description Document for Microwave Diagnostics (DDD 5.5 F) [2] released in the same year. Since that time, when the ITER design was largely complete, an official agreement between the international parties has been put in place and detailed planning and construction have begun in earnest. Some of the ideas for the port plug design and ECE instruments have changed since the DDD and a couple of design studies have been completed. Here a brief summary of these reports and other developments are given. Section 2 discusses current plans for the ECE instruments, Section 3 covers the limits of spatial resolution for ECE, Section 4 talks about the prospects for an oblique view in ITER, and finally Section 5 discusses some other issues related to ECE systems.

\section{ECE Instrument Plans}

The ECE diagnostic systems on ITER will consist of the port plug components including the front end optics, the calibration source, waveguide and windows, and the instruments, which will be of two types: a heterodyne radiometer and a Michelson interferometer. The port plug parts and instrument suite are based on those put forth in the DDD document with some significant modifications derived from recently emerged ideas and capabilities. Currently the design responsibility for these systems lies with the US and India: the US has the entirety of the port plug hardware plus one radiometer instrument and India has the other radiometer, the Michelson interferometer, and all the external waveguide including the power and polarization splitter.

The most recent conceptualization of the ECE port plug (E09) as of this writing is shown in Fig. 1. The port plug contains two views coupled to two transmission lines, the primary reason being to provide redundancy for this important diagnostic. The front ends contain Gaussian beam optics to achieve the best possible resolution in the plasma. A hot calibration source in front of the last focusing element is a part of each line. At present both lines view the plasma on a horizontal chord approximately at the plasma midplane (about $76 \mathrm{~cm}$ above the machine midplane). One of the views may be redesigned to 
view the plasma obliquely with a small toroidal angle of 10-20 degrees; the reason for doing this will be discussed in Section 4.
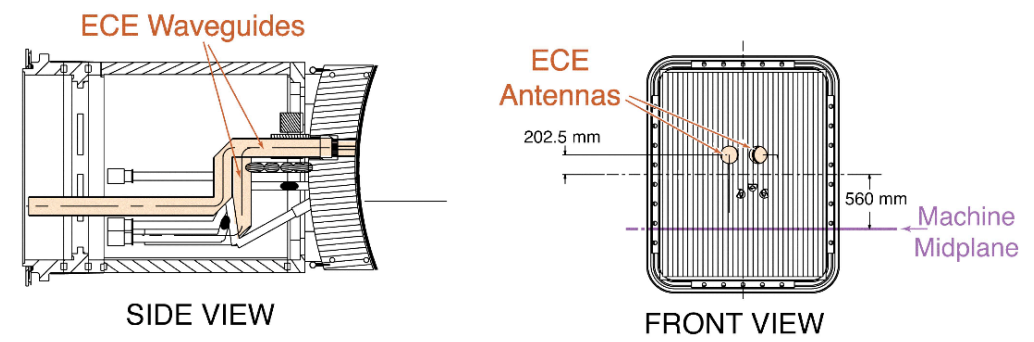

Figure 1. A conception of the ECE transmission line layout in the E09 port plug of ITER. The line at $560 \mathrm{~mm}$ is the horizontal midplane of the port plug; the midplane of the ECE antennas is $202.5 \mathrm{~mm}$ above that.

The ECE set of instruments will be comprised of two radiometers and a multichannel Michelson interferometer. Each radiometer will cover a different range of frequencies: 1) $110-220 \mathrm{GHz}$ for 1 st harmonic O-mode measurements and 2) 220-320 GHz for 2nd harmonic X-mode measurements. One small challenge will be to build the radiometer to cover the range $300-320 \mathrm{GHz}$ as local oscillators operating fundamentally above $300 \mathrm{GHz}$ with sufficient power are hard to obtain. Part of the solution would be to have a $40 \mathrm{GHz}$ wide IF as in the C-MOD radiometer [3] hence the local oscillator could be at $280 \mathrm{GHz}$, which could be achieved with a frequency-doubled $140 \mathrm{GHz}$ oscillator. The envisioned Michelson interferometer would be of the type employing a rotating helicoidal reflector as was recently constructed for JET [4]. Because it has several mirrors on the rotating wheel the interferometer can be configured with multiple channels to measure, for example, O-mode and X-mode simultaneously (one of the original design aims [1]). Frequency coverage of the Michelson would need to be up to at least $1000 \mathrm{GHz}$ to measure optically thin frequencies and evaluate wall reflectivity and electron cyclotron power losses in reactor grade plasmas.

\section{ECE spatial resolution}

An important requirement for ECE on ITER is that it achieve similar spatial resolution as in present-day machines, nominally $\sim a / 30$, where $a$ is the minor radius. The chief limiting factors to this goal are restricted physical access for antennas and relativistic broadening due to the extreme electron temperatures. 
As part of a USIPO-commissioned study a review of the possible antennas that could be used in the E09 port plug has recently been completed [5]. The starting point was the periscope layout in the conceptual design shown in Fig. 1 and three main options were considered: 1) a Gaussian telescope antenna with two focusing elements at the ends of the periscope, 2) an antenna with the first element a focusing mirror and the second element a flat turning mirror, and 3) a simple waveguide aperture located $30 \mathrm{~cm}$ behind the first wall edge. For the second case the focal length and position of the first mirror were varied to optimize the beam pattern. The beam patterns were calculated for first harmonic O-mode and second harmonic X-mode frequencies in ITER for operation at $B_{\mathrm{T}}=$ $5.3 \mathrm{~T}$.

The results of the antenna study are shown in Fig. 2. The left and right plots are the full-width half-max of the Gaussian beam versus major radius for the $1-\mathrm{O}$ and $2-\mathrm{X}$ frequencies respectively. There is a clearly superior configuration for the antenna which is one of the single ellipsoidal and flat turning mirror options. As will be discussed in the next section, due to relativistic broadening, access to the high-field side of the plasma will be limited so it is relatively unimportant if the antenna pattern diverges strongly there. Of note is that all antenna configurations have beam waists less than $10 \mathrm{~cm}$ on the low-field side, a small fraction of the vertical extent of typical discharges. One thing that came to light in this study is the flexibility of the single focusing element system; an optimal design can be found for a wide set of parameters.
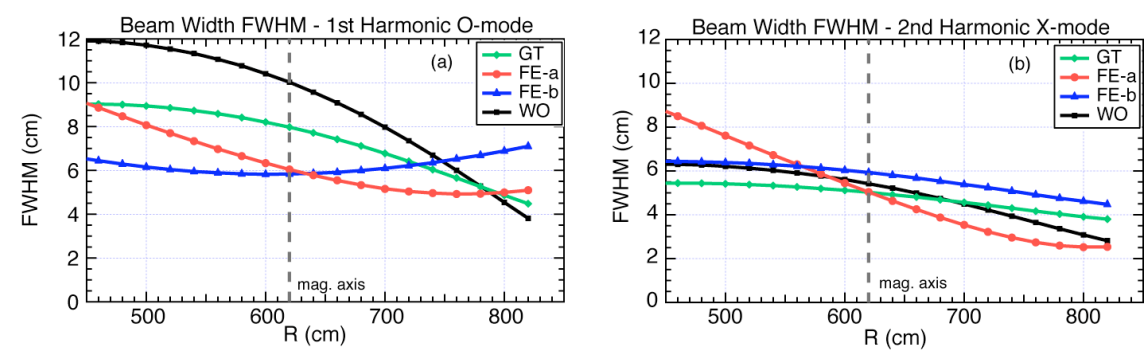

Fig. 2 (a) Beam pattern plots versus major radius for $1^{\text {st }}$ harmonic O-mode frequencies in ITER. (b) Same for $2^{\text {nd }}$ harmonic X-mode. Options shown are Gaussian Telescope (GT), Flat plus Ellipsoidal mirrors, case $a$ and $b$ (FE-a,-b), and Waveguide Only (WO).

While the beam pattern determines the poloidal and toroidal limits of the ITER ECE viewing volume, the radial extent is determined primarily by relativistic broadening. For this work an ECE simulation code was used to calculate the absorption and emission factors for an ITER plasma. The code 
ECESIM, originally developed for DIII-D, was benchmarked against the ECELS code used previously to for ITER calculations [6]. Both use standard Bornatici formulas [7] with corrections for $T_{\mathrm{e}}>10 \mathrm{keV}$. To evaluate spatial resolution the emissivity function is calculated for each frequency, harmonic, and polarization and the spatial width is determined from the distance between the $5 \%$ and $95 \%$ emission radii.

The O- and X-mode results for a Scenario 2 plasma with a central electron temperature of $25 \mathrm{keV}$ are shown in Fig. 3 and in Table 1. The two ranges of frequencies that can provide viable $T_{\mathrm{e}}$ measurements are the first harmonic Omode and second harmonic X-mode. Higher harmonics, although optically thick, have unacceptably wide emission regions. The 1-O range is preferable for the broadest coverage of the plasma. For high density cases the $2-\mathrm{X}$ range is available; although this range cannot achieve a high spatial resolution is at the plasma center, it is desirable for edge $T_{\mathrm{e}}$ measurements where it has the narrowest emission widths. A notable point is that, due to the reabsorption from strongly downshifted higher harmonics, access to the high-field side is severely limited as indicated by the shaded region of the plots.
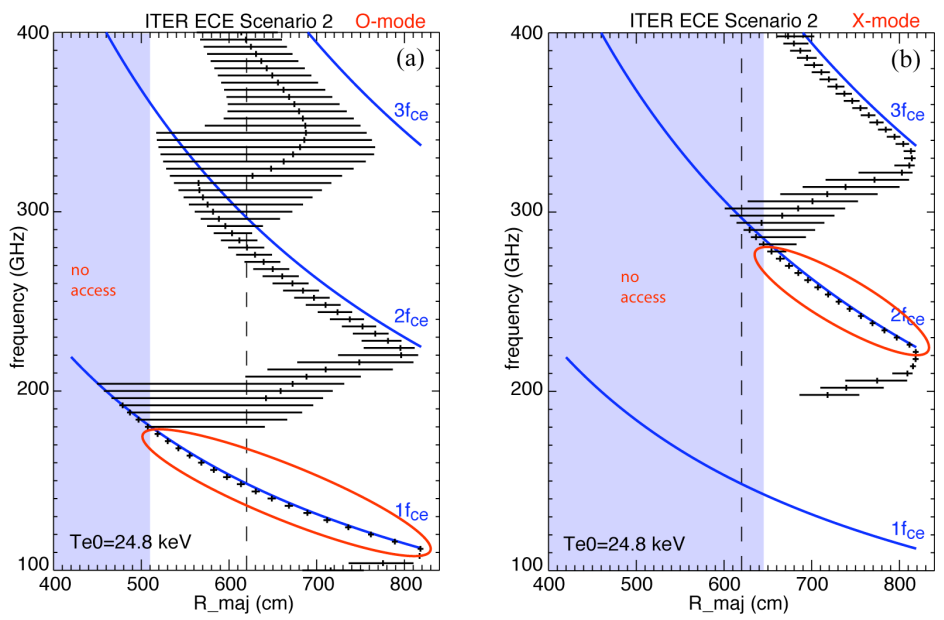

Fig. 3 Plots of ECE emission width due to relativistic broadening versus major radius for an ITER Scenario 2 plasma with $T_{\mathrm{e}}(0)=25 \mathrm{keV}$. (a) O-mode, (b) X-mode. Regions circled in red are usable frequency ranges for localized $T_{\mathrm{e}}$ measurements.

The spatial resolution requirements for ECE on ITER are $\Delta R=a / 30$ or $\sim 7 \mathrm{~cm}$. From Table 1 it can be seen that for $1-\mathrm{O}$ and $2-\mathrm{X}$ resonant locations outside of $R=730 \mathrm{~cm}$ this requirement is met. Closer to the magnetic axis the 
requirement is not met, but is still less than $10 \mathrm{~cm}$ for much of the measurable region. This is comparable to the vertical spot size of $7 \mathrm{~cm}$ or less from the optimized antenna patterns and should be sufficient to resolve many $T_{\text {e }}$ profile features and structures. For example, recent calculations of Alfvén eigenmodes on ITER predict $T_{\mathrm{e}}$ structures of $20-50 \mathrm{~cm}$ vertically and radially [8] and the ECE resolution should be sufficient to observe these.

Table 1.1 Widths of Emission Layer for 1st Harmonic O-mode, Scenario 2

\begin{tabular}{|l|c|c|c|c|c|c|c|c|c|c|}
\hline R maj $(\mathrm{cm})$ & 620 & 640 & 660 & 680 & 700 & 720 & 740 & 760 & 780 & 800 \\
\hline Freq. $(\mathrm{GHz})$ & 148 & 144 & 139 & 135 & 131 & 128 & 124 & 121 & 118 & 115 \\
\hline Width $(\mathrm{cm})$ & 8.9 & 9.2 & 9.3 & 9.1 & 8.6 & 7.9 & 7.0 & 6.2 & 5.5 & 4.8 \\
\hline
\end{tabular}

Table 1.2 Widths of Emission Layer for 2nd Harmonic X-mode, Scenario 2

\begin{tabular}{|l|c|c|c|c|c|c|c|c|c|c|}
\hline R_maj(cm) & 620 & 640 & 660 & 680 & 700 & 720 & 740 & 760 & 780 & 800 \\
\hline Freq. $(\mathrm{GHz})$ & 297 & 287 & 279 & 271 & 263 & 256 & 249 & 242 & 236 & 230 \\
\hline Width $(\mathrm{cm})$ & 114 & 67 & 27 & 8.6 & 7.8 & 6.9 & 6.0 & 5.1 & 4.4 & 3.9 \\
\hline
\end{tabular}

\section{Non-thermal ECE}

A source of unease about obtaining accurate $T_{\mathrm{e}}$ measurements from ECE on ITER is the specter of non-Maxwellian electron distributions. ITER will employ several auxiliary heating methods such as NBI, LHCD, and ECH/ECCD known to produce non-thermal electrons. Although these changes are understood and predictable in many cases, in some they are not. In particular, the phenomenon of the Thomson Scattering (TS), ECE $T_{\mathrm{e}}$ discrepancy seen on TFTR, JET and other tokamaks, where the ECE measures increasingly higher central $T_{\mathrm{e}}$ than TS in NBI-heated discharges with $T_{\mathrm{e}}(0)>7 \mathrm{keV}$, has yet to be satisfactorily explained [9].

A single perpendicular view for ECE will provide a limited amount of information on non-thermals. As is well known, several views at multiple angles can give additional data with which to narrow down the distribution function. The prospect of non-thermals in ITER has led to the suggestion of changing one of the two ECE antennas to an oblique view and the completion of a study on the ramifications of such a modification [10].

In brief summary, the report finds that an oblique-viewing ECE antenna would provide vital additional information while sacrificing little in terms of thermal $T_{\mathrm{e}}$ measurements. In case of a non-thermal distribution, $\mathrm{X}$ - and $\mathrm{O}$-mode data from a 10-20 degree oblique antenna in would reveal distortions to the distribution function in the $5-70 \mathrm{keV}$ kinetic energy range, a range where the 
TS/ECE discrepancy is thought to originate. The modifications to antenna and transmission line would easily fit in the port plug and the ECE $T_{\mathrm{e}}$ measurement would have nearly the same spatial resolution and radial coverage as the perpendicular view. As of this writing, a decision on implementing this modification to the ECE port has not been made.

\section{Other Challenges and Conclusions}

There are numerous other challenges for the ECE system, particularly in the port plug hardware. An example is the hot calibration source that lies at the end closest to the plasma. The requirement that it operate at $600^{\circ} \mathrm{C}$ and be stable and reliable for up to five years poses an engineering challenge. Work has recently been done on this and has been described in a USIPO report [5]. A material has been selected, $\mathrm{SiC}$, because of its good thermal, mechanical, and microwave absorption properties, and a design for a hot surface element comprised of regular pyramids has been chosen. Modeling shows that excellent uniformity of the surface temperature can be expected due to $\mathrm{SiC}^{\prime}$ s high thermal conductivity; temperature variations less than $6^{\circ} \mathrm{C}$ are seen. An effort is currently ongoing to find a suitable heating method for the $\mathrm{SiC}$ element; various heating sources, from IR to RF are being considered.

Other challenges are finding a suitable low-loss broadband transmission system without significant loss bands as for example due to Bragg reflection in corrugated waveguide. Also needed is a viable design for narrowband notch filters to protect the ECE instruments from ECH without sacrificing full profile access.

Overall the difficulties in developing an ECE diagnostic system for ITER are manageable and it is expected that a well-functioning system can be implemented. Although antenna access and relativistic broadening are bigger problems than on current devices, it will still be possible to achieve good spatial resolution, particularly when considered with respect to the size of the plasma. The possibility of measurements being tainted by non-thermal emission is a concern, and recommendations have been made to acquire as much information as possible to understand these cases. Clearly the ECE diagnostic on ITER will require more analysis and simulations than current systems, taking into account effects due to the extreme plasma conditions, to produce useful and accurate electron temperature information. 


\section{Acknowledgments}

The authors extend grateful thanks to A.E. Costley and G. Vayakis for helpful discussions and information. This work supported by DOE Grant FG0297ER54415 and USIPO Contract S007684-R.

\section{References}

1. G. Vayakis, D.V. Barlett, A.E. Costley, ITER JCT Home Teams, Fusion Eng. Design 53, 221 (2001).

2. Design Description Document Diagnostics 5.5.F (DDD 5.5 F) N55DDD 6 01-06-01 W 0.3. The document can be found at http://www.pppl.gov/usiter-diagnostics/Instrumentation-Packages/LowField- Side-Reflectometer/ddd 55f microwave 1.4.doc.pdf

3. J.W. Heard, et al, Rev. Sci. Instrum. 70, 1011 (1999).

4. C. Sozzi, et al, Proc. 14th Joint Workshop on ECE and ECRH, (Santorini, Greece, May 2006) ed. Avrilios Lazaros (Heliotopos Conferences Ltd., Athens, Greece, 2006,ISBN: 960-89228-2-8) p. 157.

5. M.E. Austin, et al, "Review of ITER ECE System," USIPO Report Number S0069937-F. The document can be found at http://www.pppl.gov/usiter-diagnostics/Instrumentation-

Packages/Electron-Cyclotron-Emission/S006937-F ITER ECE Report (U. Texas).pdf

6. D.V. Bartlett and H. Bindslev, in P.E. Stott et al (Eds.), Diagnostics for Experimental Thermonuclear Fusion Reactors 2, Plenum Press, New York, 1998, pp. 171-180.

7. M. Bornatici, R. Cano, O. DeBarbieri, and F. Engelmann, Nucl. Fusion 23, 1153 (1983).

8. M.A. Van Zeeland, personal communication

9. E. de la Luna, et al., Rev. Sci. Instrum, 74, 1414 (2003).

10. G. Taylor and R.W. Harvey, "ITER ECE Oblique Report (PPPL)," USIPO Report. The document can be found at http://www.pppl.gov/usiter-diagnostics/InstrumentationPackages/Electron-Cyclotron-Emission/ITER ECE Oblique Report (PPPL).doc 